\title{
Abnormal accumulation of autophagic vesicles correlates with axonal and synaptic pathology in young Alzheimer's mice hippocampus
}

\author{
Raquel Sanchez-Varo $\cdot$ Laura Trujillo-Estrada $\cdot$ Elisabeth Sanchez-Mejias $\cdot$ Manuel Torres $•$ \\ David Baglietto-Vargas · Ines Moreno-Gonzalez $\cdot$ Vanessa De Castro $\cdot$ Sebastian Jimenez \\ Diego Ruano • Marisa Vizuete $\cdot$ Jose Carlos Davila $\cdot$ Jose Manuel Garcia-Verdugo • \\ Antonio Jesus Jimenez $\cdot$ Javier Vitorica $\cdot$ Antonia Gutierrez
}

Received: 22 July 2011/Revised: 27 September 2011/Accepted: 13 October 2011/Published online: 22 October 2011

(C) The Author(s) 2011. This article is published with open access at Springerlink.com

\begin{abstract}
Dystrophic neurites associated with amyloid plaques precede neuronal death and manifest early in Alzheimer's disease (AD). In this work we have characterized the plaque-associated neuritic pathology in the hippocampus of young (4- to 6-month-old) $\mathrm{PS} 1^{\mathrm{M} 146 \mathrm{~L}}$ / $\mathrm{APP}^{751 \mathrm{SL}}$ mice model, as the initial degenerative process underlying functional disturbance prior to neuronal loss. Neuritic plaques accounted for almost all fibrillar deposits and an axonal origin of the dystrophies was demonstrated. The early induction of autophagy pathology was evidenced by increased protein levels of the autophagosome marker LC3 that was localized in the axonal dystrophies, and by
\end{abstract}

R. Sanchez-Varo $\cdot$ L. Trujillo-Estrada $\cdot$ E. Sanchez-Mejias D. Baglietto-Vargas · I. Moreno-Gonzalez · V. De Castro · J. C. Davila · A. J. Jimenez · A. Gutierrez $(\bowtie)$

Dpto. Biología Celular, Genética y Fisiología, Facultad de Ciencias, Universidad de Málaga, Campus de Teatinos, 29071 Málaga, Spain

e-mail: agutierrez@uma.es

M. Torres - S. Jimenez $\cdot$ D. Ruano $\cdot$ M. Vizuete $\cdot$ J. Vitorica Dpto. Bioquímica y Biología Molecular, Facultad de Farmacia, Universidad de Sevilla, Seville, Spain

J. M. Garcia-Verdugo

Laboratorio de Morfología Celular, Unidad Mixta Centro de Investigación Príncipe Felipe-UVEG, Valencia, Spain

R. Sanchez-Varo · L. Trujillo-Estrada · E. Sanchez-Mejias · M. Torres · D. Baglietto-Vargas · I. Moreno-Gonzalez ·

V. De Castro - S. Jimenez - D. Ruano - M. Vizuete ·

J. C. Davila - J. M. Garcia-Verdugo - J. Vitorica - A. Gutierrez Centro de Investigación Biomédica en Red sobre Enfermedades Neurodegenerativas (CIBERNED), Madrid, Spain

M. Torres - S. Jimenez $\cdot$ D. Ruano $\cdot$ M. Vizuete $\cdot$ J. Vitorica Instituto de Biomedicina de Sevilla (IBiS)-Hospital Universitario Virgen del Rocío/CSIC/Universidad de Sevilla, Seville, Spain electron microscopic identification of numerous autophagic vesicles filling and causing the axonal swellings. Early neuritic cytoskeletal defects determined by the presence of phosphorylated tau (AT8-positive) and actin-cofilin rods along with decreased levels of kinesin- 1 and dynein motor proteins could be responsible for this extensive vesicle accumulation within dystrophic neurites. Although microsomal $A \beta$ oligomers were identified, the presence of A11-immunopositive $A \beta$ plaques also suggested a direct role of plaque-associated $A \beta$ oligomers in defective axonal transport and disease progression. Most importantly, presynaptic terminals morphologically disrupted by abnormal autophagic vesicle buildup were identified ultrastructurally and further supported by synaptosome isolation. Finally, these early abnormalities in axonal and presynaptic structures might represent the morphological substrate of hippocampal dysfunction preceding synaptic and neuronal loss and could significantly contribute to $\mathrm{AD}$ pathology in the preclinical stages.

Keywords PS1/APP transgenic mice . Dystrophic neurites · Electron microscopy - LC3 . Amyloid plaques · Presynaptic terminals

\section{Introduction}

Alzheimer's disease (AD) gradually damages the function and structure of particularly vulnerable brain areas, as those used for memory and cognition. Accumulation of aggregated proteins at the extracellular (amyloid-beta, $A \beta$ ) and intracellular (hyperphosphorylated tau) levels is one of the major abnormalities found in the brain of $\mathrm{AD}$ patients (revised in [13, 26, 50]). Another key pathological feature, that defines this disease, is the early appearance of amyloid 
plaque-associated neuritic changes in the form of dystrophic neurites, together with a selective loss of connections and neuronal groups. Dystrophic neurites, defined as thickened or irregular neuronal processes, are considered to be an expression of a widespread alteration of the neuronal cytoskeleton. In $\mathrm{AD}$, dystrophic axons are particularly abundant in the hippocampal fiber systems originating from the subiculum, CA1, and the entorhinal cortex [57]. Synaptic loss in both the neocortex and the hippocampus is, so far, the best pathological correlate of early cognitive decline $[19,41,52-54,59,61]$. The initial neuritic degenerative changes may represent an early manifestation of axonal damage that precede the appearance of synaptic loss and, therefore, a promising disease-modifying morphological target for early intervention strategies to reverse the damage and rescue the deteriorating neurons. Supporting the idea that these changes could be potentially reversible, a recent study has reported that dystrophic axons surrounding amyloid plaques remain connected to viable neuronal bodies over a relatively long period of time [1].

The expression of the human amyloid precursor protein (APP) with single or double mutations in transgenic mouse lines leads to the formation of neuritic plaques with clusters of dystrophic neurites and glial recruitment that resembles the amyloid pathology seen in $\mathrm{AD}$ brains $[4,28]$. After plaque formation, neuritic abnormalities progressively develop as shown by in vivo multiphoton imaging in an AD model [42]; however, the cellular dysfunction underlying the neuritic pathology is not well understood. Numerous autophagic vacuoles accumulate within dystrophic neurites in the brains of humans with $A D$ and $A D$ models [46, 47, 66] and several lines of investigation support the notion that defects in the autophagy process, a cellular catabolic mechanism essential for the degradation of aggregated proteins and organelles, significantly contributes to $\mathrm{AD}$ pathogenesis [11, 37, 46, 48]. Interestingly, it has been reported that autophagic compartments participate in APP processing and $\mathrm{A} \beta$ peptides production $[65$, 66] suggesting a possible causal relationship between plaque formation and neuritic dystrophy. Remarkably, restoring the intracellular autophagy pathway ameliorates disease progression and cognition deficits in a transgenic model [65] proving the potential therapeutic value of autophagy induction in early stages of the disease for neuronal function recovery.

Here, in this work, we have characterized the morphological and subcellular abnormalities associated with dystrophic neurites around plaques in the hippocampus of our PS1/APP model at 4-6 months of age. Such neuritic abnormalities may result in defects in maintaining axonal and synaptic terminals structure and function. As we have reported previously, this bigenic model reproduces major amyloid-induced pathogenic steps seen in humans. The most relevant feature of our model is that, unlike many other transgenic mice in which neuronal loss is not observed, the selective neurodegenerative phenotype with specific subsets of interneurons and pyramidal neurons is affected in hippocampus and entorhinal cortex, following a regional and temporal pattern $[5,29,44,51]$. This neuronal loss was found to be associated with a neurotoxic inflammatory response induced by soluble oligomeric A $\beta$ peptides [29]. A better understanding of the pathological basis of the neuritic changes, prior to neuronal loss in this model, will provide valuable insights into the potential causes of early axonal damage and synaptic dysfunction and will further improve the accuracy of preclinical evaluation of novel therapeutic agents intended to reverse axonal damage.

\section{Materials and methods}

\section{Transgenic mice}

The generation and characterization of PS1/APP transgenic (tg) mice has been reported previously $[5,10,12,29,30$, $44,51]$. These double transgenic mice (C57BL/6 background) were obtained by crossing homozygotic PS1M146L transgenic mice with heterozygotic Thy1APP751SL (Swedish: K670N, M671L and London: V717I FAD mutations) mice (Charles River, France). Mice represented F6-F10 offspring of heterozygous transgenic mice. Non-transgenic mice of the same genetic background and ages were used as controls. All animal experiments were carried out in accordance with the European Union regulations and approved by the committee of animal use for research at Malaga University.

\section{Antibodies}

The following primary antibodies were used in this study: anti-human amyloid precursor protein (hAPP) rabbit polyclonal (1:20,000, Sigma A8717); anti-A $\beta$ (clone 6E10) mouse monoclonal (1:5,000, Sigma A1474); anti-oligomer A11 (recognizes $\mathrm{A} \beta 42$ oligomers but not monomers or fibrils) rabbit polyclonal (1:500; Millipore AB9234); anti$\mathrm{A} \beta_{42}$ rabbit polyclonal (1:40; Biosource 44-344); antiphospho-PHF-tau pSer202/Thr205 mouse monoclonal (clone AT8) (1:500; Pierce MN1020); anti-cofilin rabbit polyclonal (1:2,000, Cytoskeleton ACFLO2); anti-microtubule-associated protein 1 light chain 3 (LC3) goat polyclonal (1:1,000; Santa Cruz Biotechnology Sc16755); anti-neurofilament $150 \mathrm{kDa}$ rabbit polyclonal (1:5,000; Millipore AB1981); anti-microtubule-associated protein 2 (MAP-2); rabbit polyclonal (1:5,000; Chemicon Ab5622); anti-synaptophysin rabbit polyclonal (1:1,000; Abcam 
ab14692); anti-vesicular GABA transporter (VGAT) guinea pig polyclonal (1:5,000; Calbiochem 676780); antivesicular glutamate transporter (VGLUT1) guinea pig polyclonal (1:10,000; Millipore AB5905); anti-human A $\beta$, $\mathrm{N}$ terminus (clone 82E1) mouse monoclonal (1:1,000, IBL 10323); anti-kinesin heavy chain (clone $\mathrm{KN}-01$ ) mouse monoclonal (1:1,000, Abcam AB9097); anti-dynein, $74 \mathrm{kDa}$ (clone 74.1) mouse monoclonal (1:1,000, Millipore MAB1618).

\section{Tissue preparation}

After deep anesthesia with sodium pentobarbital $(60 \mathrm{mg} /$ $\mathrm{kg}$ ), 2-, 4- and 6-month-old control (WT), PS1 and PS1/ APP tg mice were perfused transcardially with $0.1 \mathrm{M}$ phosphate buffered saline (PBS), pH 7.4 followed by $4 \%$ paraformaldehyde, $75 \mathrm{mM}$ lysine, $10 \mathrm{mM}$ sodium metaperiodate in $0.1 \mathrm{M}$ phosphate buffer (PB), $\mathrm{pH}$ 7.4. Brains were then removed, post-fixed overnight in the same fixative at $4^{\circ} \mathrm{C}$, cryoprotected in $30 \%$ sucrose, sectioned at $40 \mu \mathrm{m}$ thickness in the coronal plane on a freezing microtome and serially collected in wells containing cold PBS and $0.02 \%$ sodium azide.

For electronic microscopy, 4.5-month-old PS1/APP tg mice were perfused transcardially with $0.1 \mathrm{M}$ phosphate buffered saline (PBS)/1\% heparin, $\mathrm{pH} 7.4$ followed by $2.5 \%$ glutaraldehyde- $2 \%$ paraformaldehyde in $0.1 \mathrm{M}$ phosphate buffer (PB), $\mathrm{pH}$ 7.4. After being removed, the brains were post-fixed in the same fixative overnight at $4^{\circ} \mathrm{C}$, washed several times with $\mathrm{PB}$, sectioned at 50 or $100 \mu \mathrm{m}$ thickness in the coronal plane on a vibratome (Leica VT1000M) and serially collected in wells containing cold PB and $0.02 \%$ sodium azide. Then, the $100-\mu \mathrm{m}$ sections were fixed in $2 \%$ osmium tetroxide in $0.1 \mathrm{M} \mathrm{PB}$ and dehydrated, to be finally embedded in Araldite (EMS, USA). Tissue blocks were cut serially into semithin $(1.5 \mu \mathrm{m})$ with a diamond knife in a Leica ultramicrotome (EM UC6), placed on slides, stained with $1 \%$ toluidine blue and explored with the light microscope for amyloid plaques. Then, selected areas from semithins were cut in ultrathin sections. Ultrathin sections were placed on Formvar-coated grids and stained with uranyl acetate and lead citrate before being examined with an electron microscope (FEI Tecnai Spirit, OR, USA).

\section{Light microscopy immunohistochemistry}

Serial sections from control (WT) and both tg mice (PS1 and PS1/APP) were processed in parallel for light microscopy immunostaining using the same batches of solutions to minimize variability in immunohistochemical labeling conditions. Free-floating sections were first treated with $3 \%$ $\mathrm{H}_{2} \mathrm{O}_{2} / 10 \%$ methanol in PBS, pH 7.4 for 20 min to inhibit endogenous peroxidases, and with avidin-biotin Blocking
Kit (Vector Labs, Burlingame, CA, USA) for $30 \mathrm{~min}$ to block endogenous avidin, biotin and biotin-binding proteins. Sections were immunoreacted with one or two of the primary antibodies over 24 or $48 \mathrm{~h}$ at room temperature. The tissuebound primary antibody was then detected by incubating for $1 \mathrm{~h}$ with the corresponding biotinylated secondary antibody (1:500 dilution, Vector Laboratories), and then followed by incubating for $90 \mathrm{~min}$ with streptavidin-conjugated horseradish peroxidase (Sigma-Aldrich) diluted 1:2,000. The peroxidase reaction was visualized with $0.05 \%$ 3-3-diaminobenzidine tetrahydrochloride (DAB, Sigma-Aldrich), $0.03 \%$ nickel ammonium sulphate and $0.01 \%$ hydrogen peroxide in PBS. After DAB, sections immunolabeled for APP, MAP-2, neurofilament or synaptophysin were incubated $3 \mathrm{~min}$ in a solution of $20 \%$ of Congo red. Sections were then mounted on gelatine-coated slides, air dried, dehydrated in graded ethanol, cleared in xylene and coverslipped with DPX (BDH) mounting medium. Specificity of the immune reactions was controlled by omitting the primary antisera.

For double immunofluorescence labelings, sections were first sequentially incubated with the indicated primaries antibodies followed by the corresponding Alexa488/568 secondary antibodies (1:1,000; Invitrogen). APP-immunolabeled sections were stained with $0.02 \%$ thioflavine-S in $50^{\circ}$ ethanol for $5 \mathrm{~min}$. Sections processed for immunofluorescence were mounted onto gelatin-coated slides, coverslipped with $0.01 \mathrm{M}$ PBS containing $50 \%$ glycerin and $3 \%$ triethylenediamine and then examined under a confocal laser microscope (Leica SP5 II).

\section{Immunoelectron microscopy}

Sections of $50 \mu \mathrm{m}$ from 4.5-month-old PS1/APP mice were first washed with PBS and incubated in a $50 \mathrm{mM}$ glycine solution $10 \mathrm{~min}$ in order to increase the antibody-binding efficiency. Following the standard immunohistochemical protocol, the tissue was incubated overnight in primary rabbit polyclonal antibodies anti-hAPP or anti-A $\beta_{42}$ in a PBS $0.1 \mathrm{M} / 0.01 \% \mathrm{Tx}-100 / 1 \%$ BSA solution at room temperature. Then, they were washed in PBS, and incubated with $1.4 \mathrm{~nm}$ gold-conjugated secondary antibody goat antirabbit IgG (1:100; Nanoprobes) for one night at room temperature. After postfixing with $1 \%$ glutaraldehyde and washing with $50 \mathrm{mM}$ sodium citrate, the labeling was enhanced with the HQ Silver ${ }^{\mathrm{TM}}$ Kit (Nanoprobes). In negative control experiments, primary antibody was omitted. Then, the slices were processed by the standard fixation, dehydration and embedding steps.

Stereological analysis

Density and size of 6E10-positive amyloid plaques were obtained by stereology-based quantification in the 
hippocampal formation of PS1/APP at 6 and 18 months of age ( $n=4$ age; 5 sections per animal) according to the optical fractionator method as previously described [44]. Briefly, an Olympus BX61 microscope and the NewCAST software package (Olympus, Glostrup, Denmark) were used. In order to obtain the plaque density, the number of plaques was quantified in five sections through the anteroposterior extent of the hippocampus and then divided between the sampled areas. CA1 subfields were defined using a $10 \times$ objective and the number of plaques was counted using a $40 \times$ objective. The number of counting frames varied with the hippocampal region or subfield layer analyzed. We used a counting frame of $7,154.7 \mu \mathrm{m}^{2}$ with step lengths of $84.58 \times 84.58 \mu \mathrm{m}$. Neurite and plaque sizes were estimated by the nucleator application with isotrophic probes ( $n=5$ radii). The number of APP-positive dystrophic neurites per plaque was quantified over Congo red stained $A \beta$ deposits. Each analysis was done by a single examiner blinded to sample identities.

\section{Total protein extraction and Western blots}

The protein pellets, obtained using the Tripure ${ }^{\mathrm{TM}}$ Isolation Reagent, were resuspended in $4 \% \mathrm{SDS}$ and $8 \mathrm{M}$ urea in $40 \mathrm{mM}$ Tris- $\mathrm{HCl}, \mathrm{pH} 7.4$ and rotated overnight at room temperature. The protein content was evaluated using Lowry.

Western blots were performed as described previously [12, 29, 51]. Briefly, 10-20 $\mu \mathrm{g}$ of protein from the different samples were loaded on $16 \%$ SDS-Tris-Tricine-PAGE and transferred to nitrocellulose (Hybond-C Extra, Amersham, Sweden). After blocking, the membranes were incubated overnight, at $4^{\circ} \mathrm{C}$, with the appropriate antibody. The membranes were then incubated with anti-mouse horseradish-peroxidase-conjugated secondary antibody (Dako, Denmark) at a dilution of 1/8,000. The blots were developed using the ECL-plus detection method (Amersham, Sweden). For quantification, the scanned (Epson 3200) images were analyzed using PCBAS program. In each experiment, the intensity of bands from WT mice and/or experimental condition were averaged and considered as 1 relative unit. Data were always normalized by the specific signal observed in 6-month-old WT group.

Synaptosomes and microsomes preparation, soluble fractions isolation and immunoprecipitation

The synaptosomal fractions were obtained basically as described previously [62]. Briefly, the tissue was homogenized (using a Dounce homogenizer) in $0.32 \mathrm{M}$ Sucrose, $10 \mathrm{mM}$ Tris- $\mathrm{HCl}$ (pH 7.4) buffer (buffer A) containing complete protease and phosphatase inhibitor cocktails (Sigma). After homogenization, the crude synaptosomal fraction (synaptosomes plus mitochondria) was isolated by two sequential centrifugations $(1,500 \times g, 10 \mathrm{~min}$ followed by $12,500 \times \mathrm{g}, 20 \mathrm{~min}$; at $\left.4^{\circ} \mathrm{C}\right)$. The crude synaptosomes were resuspended in $13 \%$ (final concentration) Ficoll 400 (in buffer A) and layered on the bottom of a discontinuous gradient, composed by buffer $\mathrm{A}$ and $7 \%$ Ficoll (in buffer A). The gradients were centrifuged at $100,000 \times g(45 \mathrm{~min}$ at $4{ }^{\circ} \mathrm{C}$ ) and the synaptosomes were isolated at the $7.5-13 \%$ interface. After washing (twice with buffer A), the protein content of the synaptosomal fractions was quantified by Lowry.

The soluble and microsomal fractions (supernatant and pellet, respectively) from PS1/APP and WT mice were obtained after centrifugation at $100,000 \times g\left(1 \mathrm{~h}, 4^{\circ} \mathrm{C}\right)$ as described previously [29, 30].

The A11 or $6 \mathrm{E} 10$ immunoprecipitation experiments were also performed as described in detail previously [29, 30]. Since the epitope recognized by A11 was sensitive to detergents, synaptosomes and microsomes were disturbed by sonication ( 4 pulses at $100 \mathrm{~W}, 30 \mathrm{~s}$ at $4^{\circ} \mathrm{C}$ ). After sonication, the synaptosomes and microsomes were centrifuged $\left(30,000 \times g, 30 \mathrm{~min}\right.$ at $\left.4^{\circ} \mathrm{C}\right)$ and soluble proteins were used for immunoprecipitation. A11 and $6 \mathrm{E} 10$ immunoprecipitation was done using $50 \mu \mathrm{g}$ of soluble protein.

\section{Statistical analysis}

Data was expressed as mean $\pm \mathrm{SD}$. The comparison between two mice groups (WT and PS1/APP mice or PS1 and PS1/APP tg mice) was done by two-tailed $t$ test, and for comparing several groups (WT, PS1 and PS1/APP mice) and ages we used one-way ANOVA, followed by Bonferroni post hoc multiple comparison test (SigmaStat ${ }^{\circledR}$ 2.03 , SPSS Inc). For both tests, the significance was set at $95 \%$ of confidence.

\section{Results}

Amyloid plaque-associated dystrophic neurites display a massive accumulation of autophagic vesicles from early ages

This PS1/APP transgenic model exhibited extracellular A $\beta$ deposits throughout the hippocampus from a very early age as illustrated in Fig. 1a with Congo red staining at 4 months. The number and size of the amyloid deposits progressively increased with age (Fig. 1b). In young mice (4- to 6-month old), the most abundant plaques were those less than $500 \mu^{2} \quad(70.53 \pm 9.74 \%)$, whereas in older mice (18 months) the vast majority of plaques $(69.41 \pm 11.73 \%)$ were medium to large $\left(>500 \mu \mathrm{m}^{2}\right)$. 
Fig. 1 Early plaque-associated neuritic dystrophy pathology in PS1/APP hippocampus. a and b APP-immunolabeled sections counterstained with Congo red for fibrillar amyloid deposits at 4 (a) and 6 (b) months of age showing the early occurrence of the neuritic pathology. The number of neuritic plaques progressively increases with age. APP-positive dystrophic neurites arise from glutamatergic neurons since the human APP transgene is exclusively expressed by principal neurons as shown in the inset. c A representative neuritic plaque formed by a core of congophilic fibrillar amyloid surrounded by numerous APPpositive dystrophic neurites. d Confocal image showing APP-positive dystrophic neurites (red) around a plaque stained with thioflavin-S (green). e Stereological quantification of the dystrophic neurites around plaques. The number of dystrophies/plaque increased with the size of plaque $\left(\mu \mathrm{m}^{2}\right)$. Data are expressed as mean $\pm \mathrm{SD}, * p<0.05$. Scale bars $\mathbf{a}$ and $\mathbf{b} 500 \mu \mathrm{m}$, inset $25 \mu \mathrm{m}$, c and $\mathbf{d} 10 \mu \mathrm{m}$. CA1-CA3 subfields of the hippocampus proper, $D G$ dentate gyrus
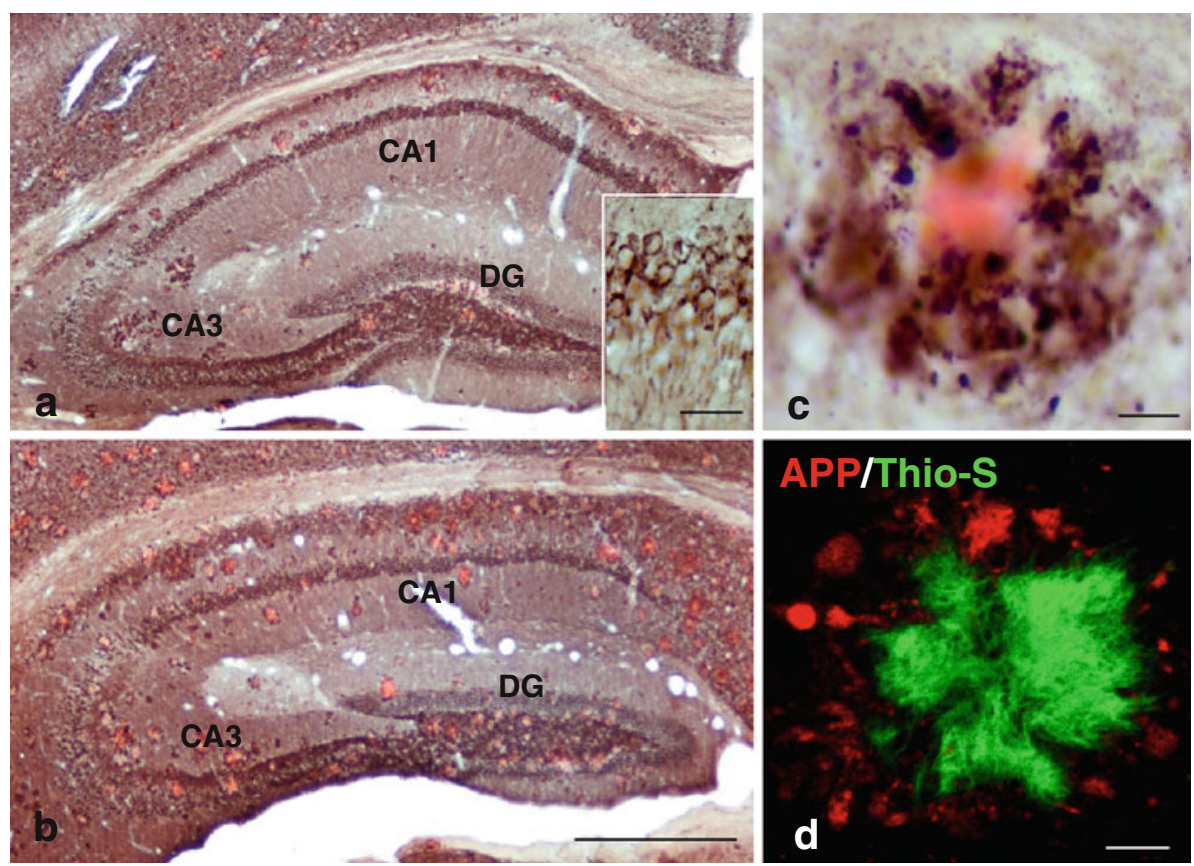

e

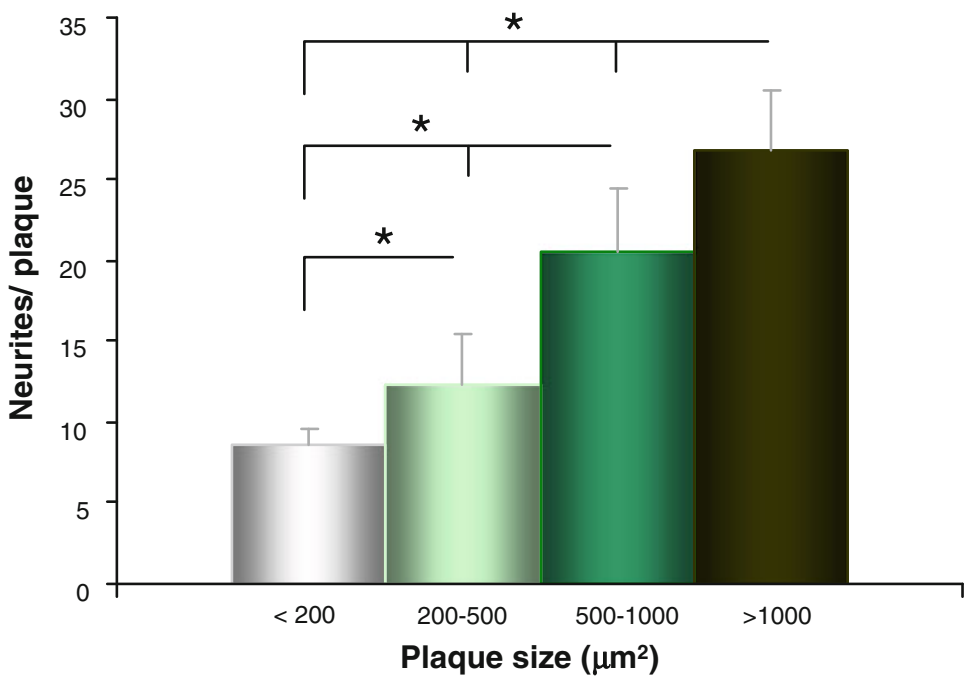

Double labeling APP/Congo red (Fig. 1a-c) and APP/ thioflavin-S (Fig. 1d) experiments demonstrated that, at every age examined, almost all $(91.61 \pm 0.14 \%$, the percentage was practically identical at 4, 6 and 18 months of age) fibrillar amyloid deposits were decorated with clusters of APP-positive dystrophic neurites (APP is a well-reported marker for dystrophies) from the time of the appearance of amyloid plaques. The number of dystrophic neurites per plaque increased with age in parallel with the size of the plaque (Fig. 1e). Results showed that, in fact, the number of these dystrophic neurites correlated with the size of the plaque and was independent of the age of the mice. Thus, neuronal pathology in the form of dystrophic neurites occurred very early in this transgenic model. These pathological structures were not found in wild-type (WT) or
PS1 transgenic mice of the same age (data not shown). Therefore, plaque-associated abnormal swelling of neuronal processes represented an early indicator of disease development and might compromise neuronal integrity and hippocampal function in young PS1/APP mice. No dystrophic neurites were found in areas remote from $A \beta$ plaques or in 2-month-old PS1/APP mice (before the A $\beta$ deposition).

Transmission electron microscopy analysis of the hippocampus of 4.5-month-old PS1/APP mice revealed a close spatial association between amyloid plaques and neuronal dystrophies (Fig. 2a, b). No dystrophic neurites were found in areas remote from plaques. These abnormal swollen neurites had a round/oval profile and were giantsized, compared to normal neuronal processes in the 


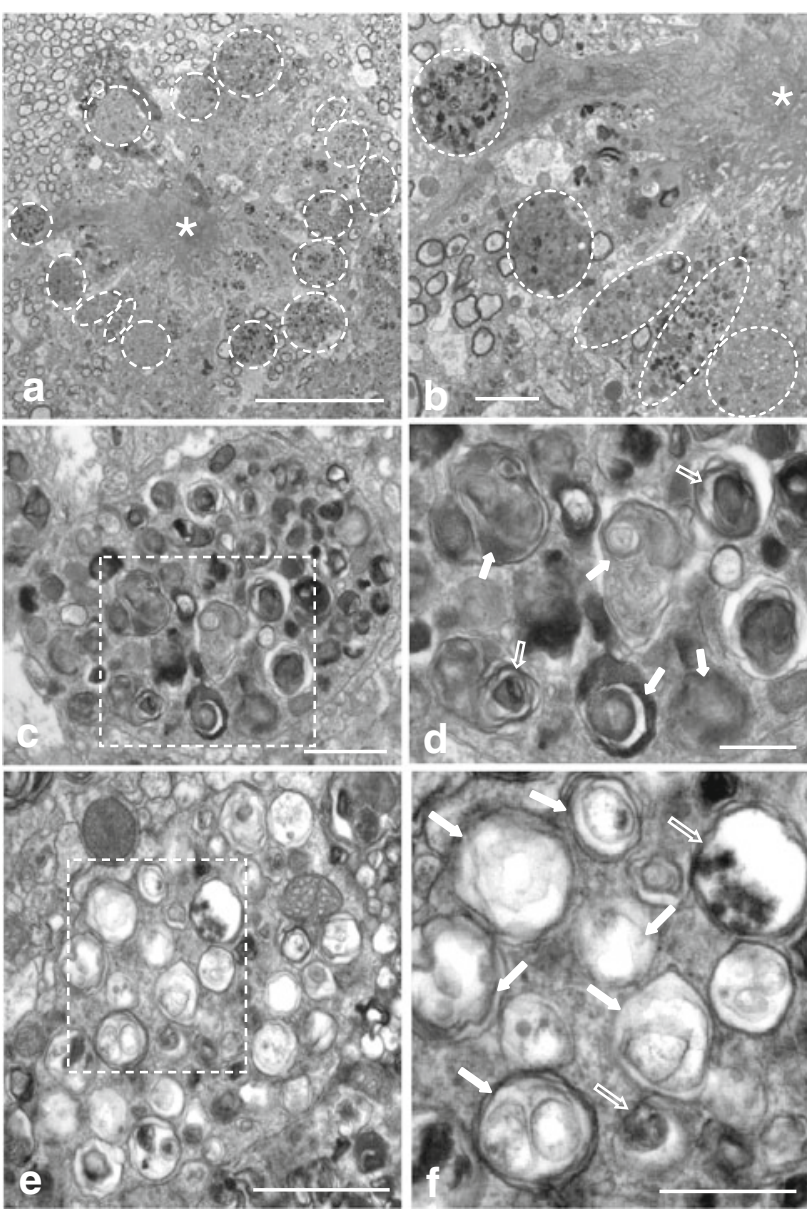

Fig. 2 Extensive accumulation of autophagic vesicles within dystrophic neurites around amyloid plaques. Transmission electron microscopy images of plaque-associated aberrant neurites in young PS1/APP hippocampus. a Low magnification image of an amyloid plaque (asterisk) surrounded by dystrophic neurites (discontinuous white circles). b A diversity of dystrophic neurites based on their subcellular content can be identified. $\mathbf{c}$ and $\mathbf{d}$ Dystrophic neurite filled with heterogeneous electrodense double-membrane vesicles (white square magnified in d) belonging to the early degrading autophagylysosomal pathway (autophagosomes); these AVs had a dense compacted amorphous (filled white arrows) or multilamellar content (empty white arrows). e and f Dystrophic neurite filled with autophagic vesicles (white square magnified in f) of distinct morphologies showing translucent (filled white arrows) or amorphous electrodense material (empty white arrows) which might represent late autophagic vesicles (autophagolysosomes). Scale bars a $10 \mu \mathrm{m}$, b $2 \mu \mathrm{m}$, c and e $1 \mu \mathrm{m}$, d and $\mathbf{f} 500 \mathrm{~nm}$

adjacent neuropil. Ultrastructural morphometric analysis (100 aberrant neurites; $n=3$ ) revealed that the predominant size of these dystrophic structures was between 10 and $50 \mathrm{\mu m}^{2}$ (63.74\%), followed by those ranging from 50 to $100 \mu \mathrm{m}^{2}(20.43 \%)$. Notably, $5 \%$ of the dystrophic neurites measured over $100 \mu \mathrm{m}^{2}$, only $9.68 \%$ of neurites were in the range $5-10 \mu \mathrm{m}^{2}$ and just $1 \%$ under $5 \mu \mathrm{m}^{2}$. In contrast, normal non-dystrophic neurites had an average size of $1.42 \pm 0.77 \mu \mathrm{m}^{2}$.
Dystrophic neurites were massively filled with collections of vacuolar structures of putative autophagic nature with different morphologies and heterogeneous intraluminal contents (Fig. 2c, e). The most common morphology corresponded to autophagic vesicles (AVs) consisting of double membrane-bound vesicles with densely compacted amorphous or multilamellar contents named as autophagosomes (Fig. 2c, d). These AVs represent the initial stages of autophagy which contain undigested compacted organellar material. In addition, there were also single or double membrane vesicles with translucent or amorphous electron-dense material in some dystrophic neurites (Fig. 2e, f), and these might presumably represent autophagosomes with partially digested material and/or the mature degradative forms of AVs (autophagolysosomes). Overall, a substantial accumulation of early and, to a less extent, late AVs within hippocampal aberrant neurites, surrounding amyloid plaques, occurred at very early ages in these PS1/APP mice.

LC3-positive autophagic vesicles within dystrophic neurites are implicated in the amyloidogenic pathway

To corroborate the autophagic nature of the heterogeneous vesicles accumulated within the dystrophic neurites, we immunostained PS1/APP hippocampal sections with the anti-LC3 antibody, a marker of autophagy (Fig. 3a, b). At 4 months (Fig. 3a), LC3 immunoreactivity was mainly found in pyramidal somata and their apical dendrites, as well as in punctate structures resembling dystrophic neurites around plaques (see insets in Fig. 3a). At 6 months (Fig. 3b), the immunoreactivity for LC3 around plaques was markedly increased while, in parallel, the staining of somata and apical dendrites decreased.

To more specifically determine the proportion of LC3 that was in the LC3-II form, which migrates faster than LC3-I on SDS-PAGE and is the form associated (by lipidation) with the autophagosomal structures, we performed quantitative immunoblot analysis of LC3-I and LC3-II forms in the hippocampus of 6-month-old PS1/APP and WT mice (Fig. 3c). Significantly higher levels of LC3-II were observed in PS1/APP mice than in age-matched WT mice $(2.15 \pm 0.35$ fold, $n=6, p<0.05)$.

Confocal imaging of double APP/LC3 immunolabeling (Fig. 3d1-d3) revealed the punctate nature of the LC3 labeling (see inset in Fig. 3d2) and the colocalization of LC3 in both APP-positive (glutamatergic) and non-APP (likely GABAergic or cholinergic) dystrophic neurites (Fig. 3d3). Considering the colocalization of APP and LC3 within dystrophic neurites in our AD model, we next wanted to assess the early implication of AVs in APP processing, and in turn likely involvement in $\mathrm{A} \beta$ production. To that end, we performed immunoelectron 
microscopy for APP and A $\beta_{42}$ in 4.5-month-old PS1/APP hippocampus. Silver-enhanced immunogold labeling revealed that APP localized preferentially to the AVs within plaque-associated dystrophic neurites (Fig. 3e-g), as well as to the Golgi and endoplasmic reticulum (ER) membranes in the neuronal cell somata (Fig. 3h). No APPlabeling was found in other organelles or plasma membrane. Immunoelectron microscopy detection of $A \beta$ was much less stronger than of APP, since optimal intracellular labeling with the antibodies for $A \beta$ forms in our model requires pre-treatment with formic acid which is not compatible with EM processing. Nevertheless, as expected and in contrast to APP immunolabeling, $A \beta_{42}$ label was mainly associated with plaques (asterisk in Fig. 3i). Interestingly, some autophagic vesicles within dystrophic neurites were also positive for the $A \beta_{42}$ antibody (inset in Fig. 3i).

Dystrophic neurites represent axonal structures with cytoskeletal abnormalities

To determine the dendritic and/or axonal nature of the plaque-associated dystrophic neurites in our PS1/APP model, we have performed light and electron microscopy studies in 4- to 6-month-old mice. Immunolabeling for the MAP-2 protein (a marker of dendritic processes) (Fig. 4a, b) and for the postsynaptic marker $\alpha 1 \mathrm{GABA}_{\mathrm{A}} \mathrm{R}$ (not shown) revealed no positive dystrophic neurites around plaques at the early ages investigated. Moreover, confocal double MAP-2/APP immunofluorescence labeling confirmed the lack of colocalization for the dendritic marker in APP-positive dystrophic neurites (Fig. 4c1-c3). On the other hand, the close spatial relationship between amyloid plaques and axonal fibers tracts in the hippocampus, as revealed by neurofilament (NF) immunolabeling and Congo red staining (Fig. 4d), along with the presence of swollen NF-positive neurites (insets in Fig. 4d) indicated a possible axonal/synaptic origin of these dystrophic structures.

In fact, the labeling of the dystrophic neurites was very patent with the presynaptic marker synaptophysin (Fig. 4e). Numerous synaptophysin-positive punctated structures were observed around amyloid plaques. To determine the neurochemical nature of these synaptophysin-positive dystrophic neurites, we performed double immunofluorescence labeling for APP and the two major neurotransmitter vesicular transporters, VGLUT1 for glutamate (Fig. 4f1-f3) and VGAT for GABA (Fig. 4g1-g3). As shown in Fig. 4f3 most APP-positive dystrophic neurites contained VGLUT1 indicating the glutamatergic nature of the abnormal axons surrounding amyloid plaques. Consistent with the exclusive expression of the human mutated transgene for APP by principal cells, many enlarged inhibitory GABAergic dystrophic neurites, immunonegative for APP, were also identified around the plaques (Fig. 4g3).

In addition, electron microscopy in the hippocampus of 4.5-month-old PS1/APP mice confirmed the presence of some dystrophic myelinated axons around/near plaques (Fig. 4h-j). These axonal dystrophies had a severe (Fig. 4h, i) to moderately (not shown) pathological number of autophagic vesicles. The enlarged size of an aberrant axon $\left(110.22 \mu \mathrm{m}^{2}\right)$ compared to adjacent normal ones $\left(1.57 \pm 0.63 \mu \mathrm{m}^{2}\right)$ is shown in Fig. $4 \mathrm{j}$.

In order to identify possible early microtubule-associated axonal transport deficits in the PS1/APP hippocampus, which might lead to vesicle accumulation (autophagic, synaptic, etc.) along axons and the consequent development of dystrophy, we first assessed tau abnormalities by quantitative Western blots experiments with the AT8 antibody (which detects tau phosphorylated at both serine 202 and threonine 205 residues, one of the first to be phosphorylated) (Fig. 5a). Immunoblotting revealed a significantly higher level of expression in young PS1/APP mice $(1.75 \pm 0.15$ fold) compared to age-matched controls. We have also confirmed by AT8 immunohistochemistry the presence of phospho-tau positive neurites surrounding amyloid plaques in 4- to 6-month-old transgenic animals (Fig. 5b). To determine whether phospho-tau was present within APPpositive dystrophic neurites, we performed double APP/AT8 immunofluorescence labeling (Fig. 5c1-c4 and d1-d4). The presence of AT8 was found in some, but not all, APP-positive dystrophic neurites (Fig. 5c3 and detail in c4; Fig. 5d3 and detail in $\mathrm{d} 4$ ).

Tau could also induce changes in the organization and stability of neuronal actin filaments, and it is known that the formation of cofilin/actin pathological bundles occludes neurites and vesicle transport. To assess possible early alterations of the actin cystoskeleton in our AD model we analyzed actin and cofilin immunolabeling in the hippocampus of young PS1/APP mice. Results showed numerous rod-like inclusions around amyloid plaques as shown for cofilin in Fig. 5e. Moreover, double APP/cofilin labeling (Fig. 5f1-f3) showed colocalization of both markers in some, particularly small, APP-positive neurites.

Finally, to further explore whether microtubule vesicular transport was compromised in young PS1/APP mice we measured the levels of kinesin-1 and dynein, two microtubule-associated motor proteins, in hippocampal protein extracts prepared from 6-month-old PS1/APP and WT mice (Fig. $5 \mathrm{~g}$ ). We found significantly lower levels of both kinesin-1 heavy chain $(-24.71 \pm 11.80 \%, n=8)$ and dynein $(-38.06 \pm 14.40 \%, n=8)$ in PS1/APP than agematched WT animals.

These microtubule, actin cytoskeletal and molecular motor defects are early pathogenic events in our AD model 

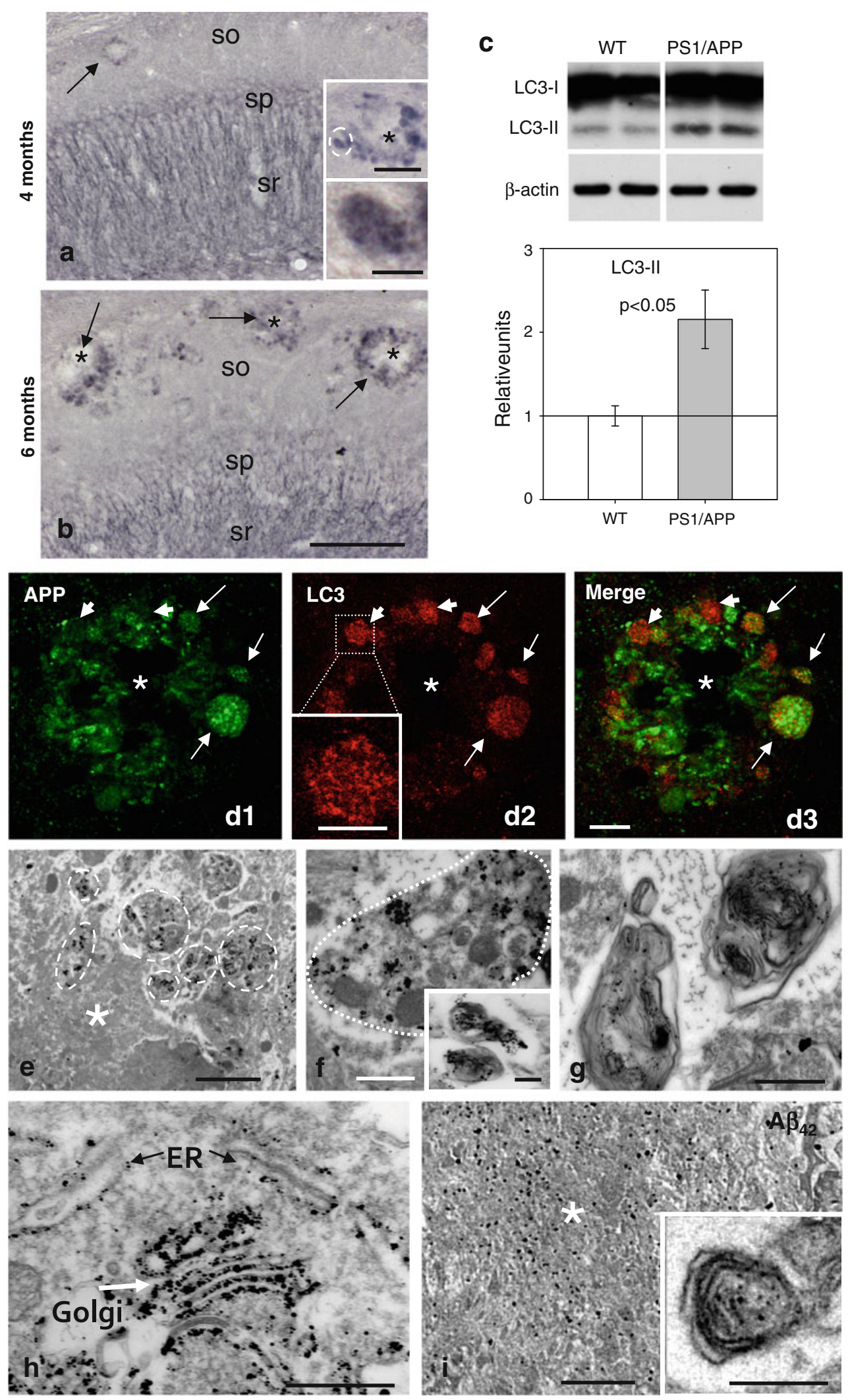
4 Fig. 3 Autophagic compartments within dystrophic neurites are sites of APP processing. $\mathbf{a}$ and $\mathbf{b}$ Immunohistochemistry for the autophagosome marker LC3 in CA1 subfield of PS1/APP mice hippocampus. At 4 months (a), immunoreactivity was concentrated in pyramidal cell somata (stratum pyramidale) and their apical dendrites (stratum radiatum). LC3-positive dystrophic neurites were surrounded amyloid plaques (upper inset in a) and were punctate in nature (the lower inset shows a high magnification image of the LC3positive dystrophy circled in the upper inset). At 6 months (b), the immunoreaction was mainly associated with dystrophic neurites around plaques (arrows) as shown here for stratum oriens (SO). c Quantitative immunoblot analysis of LC3-II form in the hippocampus of 6-month-old PS1/APP and WT mice. PS1/APP mice had significantly higher LC3-II levels than age-matched WT mice $(n=6$, $p<0.05)$. d1-d3 Confocal images of double immunofluorescence labeling for APP (green) and LC3 (red); APP-positive dystrophic neurites around an amyloid plaque (asterisk) displayed the LC3 marker (long arrows); some LC3-positive dystrophic neurites were negative for APP (short arrows) indicating the non-glutamatergic origin of these aberrant neurites. e Dystrophic neurites (encircled) around amyloid plaques (asterisk) were APP immunopositive as revealed by electron microscopy using silver-enhanced immunogold labeling. f Higher magnification of a dystrophic neurite immunopositive for APP; small open squares indicate mitochondria; inset shows two autophagosomes immunogold-labeled for APP. g Higher magnification image of two autophagic vesicles immunogold-labeled for APP. $\mathbf{h}$ In addition to autophagic vesicles, APP immunogold labeling was associated with the endoplasmic reticulum $(E R)$ and Golgi cisternae in neuronal somata. i Immunogold labeling revealed that A $\beta 42$ peptides localize to amyloid plaques (asterisk) and autophagic vesicles (inset). so stratum oriens, $s p$ stratum pyramidale, $s r$ stratum radiatum. Scale bars a and b $100 \mu \mathrm{m}$ (upper inset in a $50 \mu \mathrm{m}$, lower inset in a $10 \mu \mathrm{m}$ ); d1-d3 $100 \mu \mathrm{m}$ (inset in $\mathbf{d 2} 2.5 \mu \mathrm{m}$ ), $\mathbf{e}-\mathbf{h} 500 \mathrm{~nm}$, inset in f $200 \mathrm{~nm}, \mathbf{i} 2 \mu \mathrm{m}$ and inset $400 \mathrm{~nm}$

and might lead to transport abnormalities and the accumulation of organelles (synaptic vesicles, autophagosomes, mitochondria, lysosomes) within axonal neurites promoting the dystrophic process.

The early axonal pathology includes morphologically disrupted presynaptic terminals

The axonal defects in our AD model could also affect presynaptic terminals. Therefore, in order to investigate the possible early synaptic pathology we examined the hippocampus of 4.5-month-old PS1/APP mice using electron microscopy (Fig. 6a-e). Our ultrastructural study showed that, near to amyloid plaques, there were presynaptic elements that displayed pathological changes including large diameter with a considerate number of AVs and, in contrast, fewer synaptic vesicles (Fig. 6a, b; presynaptic terminals outlined with a white line). The presence of presynaptic terminals, at the beginning of the dystrophy process, with few and early stage AVs formation, as well as with synaptic-like vesicles were also detected (Fig. 6c-e; see also the presynaptic terminal outlined with a black line in Fig. 6b). However, these altered presynaptic elements were making synaptic contacts with morphologically normal dendrites or dendritic spines with postsynaptic density (see Fig. 6a-d). These morphologically altered presynaptic terminals may represent the initial stages of synaptic disruption and loss.

Moreover, we compared the LC3-II accumulation in synaptosomal and microsomal fractions isolated from 6-month-old WT and PS1/APP animals (Fig. 6f). As expected, a low percentage of LC3-II was observed in isolated synaptosomes from WT mice (15.42 $\pm 2.25 \%$, $n=4$, of microsomal fractions in WT mice). Further, in agreement with our electron microscopy studies, the amount of LC3-II in PS1/APP mice was higher in both synaptosomal and microsomal fractions. Although the relative abundance of LC3-II presented in PS1/APPderived synaptosomes was still low (22.22 $\pm 3.84 \%$ of PS1/APP microsomal LC3-II), the level was consistently higher $(2.54 \pm 0.54, n=5)$ than in WT synaptosomes.

These data demonstrated the existence of an early autophagy-associated axonal/synaptic pathology in the hippocampus of this AD mouse model.

Extracellular periplaque oligomeric $A \beta$ spatially correlates with axonal/synaptic dystrophy

Taking into account the close spatial relationship between axonal dystrophies and $A \beta$ plaques, we next examined the possible intracellular and/or extracellular origin of the pathogenic $A \beta$ agent. We first investigated the possible intracellular accumulation of oligomeric $A \beta$ in isolated synaptosomes and microsomes by immunoprecipitation experiments using the monoclonal antibody 6E10 (Fig. 7a). Results demonstrated the presence of a relatively large accumulation of monomeric $\mathrm{A} \beta$ in synaptosomes, whereas lower levels were detected in microsomes (Fig. 7a1). Furthermore, within the different $A \beta$ peptides, the $A \beta_{42}$ was the major form observed in these synaptosomal fractions (Fig. 7a2). On the other hand, no $A \beta$ oligomers were observed with this approach.

We have also used the anti-oligomeric A $\beta$ antibody A11 in immunohistochemistry and immunoprecipitation experiments. Our results demonstrated that most $A \beta$ plaques were immunopositive for the A11 antibody (Fig. 7b), with a preferential immunolabeling at the plaque periphery (see inset). Furthermore, A11-immunoprecipitation using the soluble (S1) fractions demonstrated the presence of minute amount of extracellular oligomeric $\mathrm{A} \beta$ (Fig. 7c) in the hippocampus of the PS1/APP mice at this early age (6 months). These data were consistent with previous experiments (see also [29, 30]). However, in spite of the relative abundance of monomeric A $\beta$ in synaptosomes, under the present experimental conditions, no $A \beta$ oligomers 


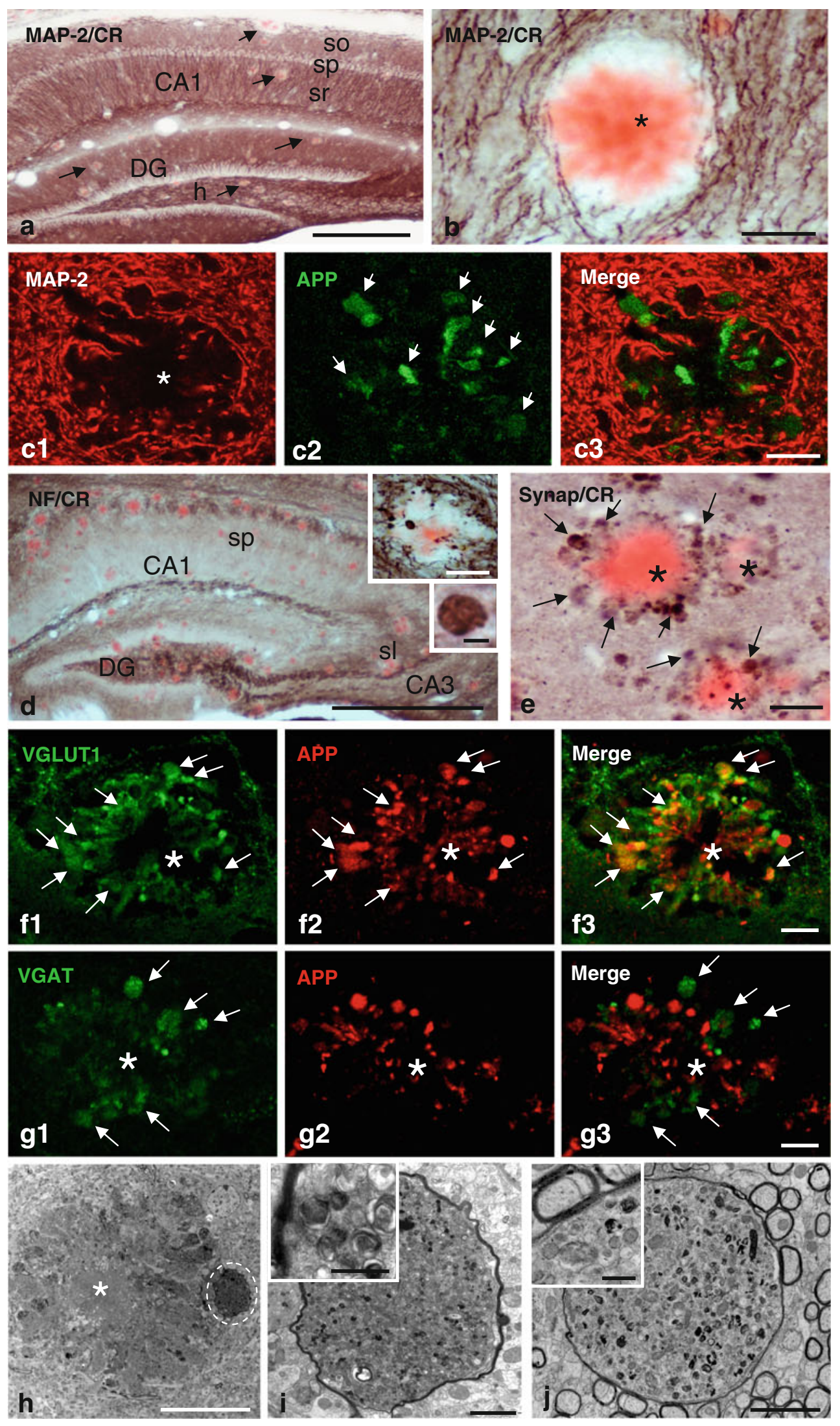


4 Fig. 4 Dystrophic neurites are of axonal origin. a MAP-2 (dendritic marker) and Congo red staining in the hippocampus of 6-month-old PS1/APP mice (arrows indicate amyloid plaques). b No dystrophic neurites immunopositive for MAP-2 were seen around amyloid plaques, as illustrated here for a Congo red stained amyloid plaque (asterisk) embedded in a MAP2-positive neuropil in the radiatum layer. c1-c3 Confocal double immunofluorescence labeling for MAP2 (red) and APP (green) revealed no colocalization of the two markers (asterisk indicates an amyloid plaque). d Strong overlapping in the location of hippocampal axonal pathways and amyloid plaques as shown here using neurofilament (NF) immunolabeling and Congo red staining. Axonal dystrophies were observed around congophilic plaques as shown in the larger inset, and a detail of an axonal swelling is shown in the smaller inset. e Neuritic dystrophies around plaques (asterisk) were immunopositive for synaptophysin (arrows). f1-f3 Confocal laser double immunofluorescence labeling for APP (red) and VGLUT1 (green) shows extensive colocalization (arrows) of the two markers indicating the axonal glutamatergic nature of APPpositive dystrophic neurites around plaques (asterisk). g1-g3 Doublelabel immunofluorescence confocal laser microscopy for APP (red) and VGAT (green) shows the existence of GABAergic axonal dystrophies (VGAT-positive and APP-negative; arrows) around amyloid plaques (asterisk). $\mathbf{h}-\mathbf{j}$ Transmission electron microscopy images. $\mathbf{h}$ Low magnification image of an amyloid plaque (asterisk) surrounded by dystrophic neurites. i At a higher magnification, detail of the dystrophic myelinated axon circled in $\mathbf{h}$; the inset shows the autophagic vesicle content of this axonal swelling and the myelin sheath. j Another representative image of a myelinated axonal swelling, filled with autophagic vesicles. The giant size of this dystrophy could be estimated by comparison with the adjacent normal myelinated axonal profiles; inset shows a detail of this axonal dystrophy. CA1-CA3 subfields of the hippocampus proper. $D G$ dentate gyrus, $s p$ stratum pyramidale, $s l$ stratum lucidum. Scale bars a $250 \mu \mathrm{m}, \mathbf{b} 25 \mu \mathrm{m}, \mathbf{c 1}-\mathbf{c 3} 15 \mu \mathrm{m}, \mathbf{d} 500 \mu \mathrm{m}$ (larger inset $50 \mu \mathrm{m}$ and smaller inset $5 \mu \mathrm{m})$, e $20 \mu \mathrm{m}$, f1-f3 and g1-g3 $10 \mu \mathrm{m}$, h $20 \mu \mathrm{m}$, i $2 \mu \mathrm{m}$ (inset $200 \mathrm{~nm}$ ), j $2 \mu \mathrm{m}$ (inset $500 \mathrm{~nm}$ )

were detected in this fraction (Fig. 7c). On the other hand, in the microsomal fractions, in the A11 immunoprecipitation experiments many non-specific bands were observed. The presence of this non-specific immunoprecipitation precluded the identification of putative oligomers. It is possible that the relative high abundance of AVs in this fraction could interfere with the immunoprecipitation experiments. Aiming to overcome this problem, the presence of the possible oligomers was also assayed using Western blots with 6E10 and 82E1 antibodies. The 6E10 antibody produced no specific signal (not shown); the 82E1 antibody, however, produced a clear specific (as compared with age-matched WT results) identification of $A \beta$ oligomers (Fig. 7d). Taken together, these data indicate the presence of $\mathrm{A} \beta$ monomers in synaptosomes and $\mathrm{A} \beta$ oligomers in extracellular plaques and the microsomal fractions.

\section{Discussion}

Here we report data showing that amyloid plaques are associated with dystrophies of axonal origin (loaded with autophagic vesicles) and constitute a very early pathological event in the hippocampus of PS1 $1^{\mathrm{M} 146 \mathrm{~L}} / \mathrm{APP}^{751 \mathrm{SL}}$ mice. The presence of morphologically disrupted presynaptic terminals may be one of the initial stages for synaptic loss and dysfunction, so far the best correlate for early symptoms in $\mathrm{AD}$ patients $[3,9,19,41,45,52,53,59,61]$ and, therefore, a pre-clinical manifestation of progression of the disease.

Amyloid accumulation is considered a key event in $\mathrm{AD}$ pathology by causing glial activation, neuritic alteration, synaptic damage and neuronal death $[34,35,42]$. All the fibrillar amyloid deposits in the hippocampus of our transgenic mice were identified as human-like neuritic plaques with dystrophic neurites and reactive gliosis. Dystrophic neurites are classically associated with compacted plaques in AD tissue [21, 24, 25, 39, 40, 57, 58] and these neuritic plaques have been considered to be a pathological correlation of dementia in $\mathrm{AD}$ patients [43]. Though neuritic dystrophy may apply to both dendritic and axonal morphological changes, in our model the predominant axonal nature of the dystrophies was demonstrated by colocalization with common axonal/synaptic (neurofilament, synaptophysin, VGLUT1 and VGAT) but not dendritic (MAP-2, $\left.\alpha 1 \mathrm{GABA}_{\mathrm{A}} \mathrm{R}\right)$ markers. Furthermore, assessment of the dystrophies using electron microscopy also confirmed their axonal structure. Notably, a thorough examination of dystrophic neurites at the electron microscopic level revealed that they were not postsynaptic to any presynaptic bouton (not shown in results). Although we cannot rule out the existence of dendritic alterations, in our model most dystrophies displayed an axonal origin. The presence of axonal dystrophies has been reported in AD patients in both early and late stages of the disease [7, 21, 22, 57] and also in another AD models $[1,8,14,20,49,64]$.

As found in the brains of people with $\operatorname{AD}[47,66]$ and in other transgenic models [65, 66], in our PS1/APP model the axonal dystrophies were seen to have a large accumulation of a great variety of vesicles in the process of autophagy. Autophagy, an efficient cellular degradation and maintenance pathway for multiple components, is not usually observed in normal brains. Recently, it has been demonstrated that PS1 mutations accelerate the pathogenesis of $\mathrm{AD}$ by impairing autophagy and organelle turnover, since PS1 is essential for autolysosome acidification and maturation [37]. The critical contribution of defective autophagy proteolytic clearance to the $\mathrm{A} \beta$ pathology has been recently demonstrated in TgCRND8 transgenic mice [65]. Restoring the autophagy-lysosomal pathway by deletion of cystatinB in these $\mathrm{AD}$ mice reduced intracellular and extracellular amyloid load, and rescued memory performance. The existence of autophagic pathology in hippocampal dystrophic neurites in our PS1/APP model was evidenced at early ages 

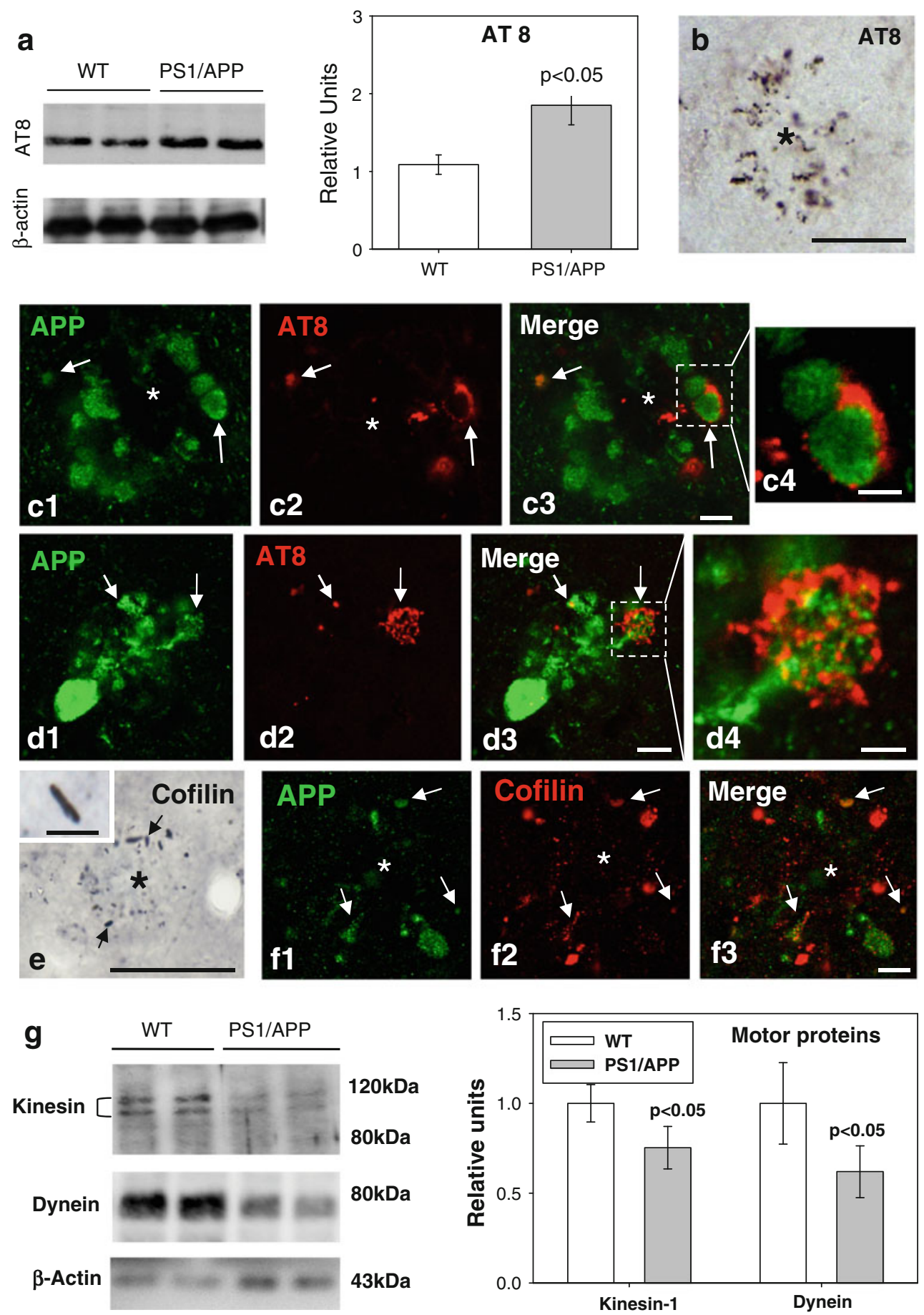

Fig. 5 Cytoskeletal pathology in young PS1/APP hippocampus. a Quantitative Western blotting using AT8 antibody in the hippocampus of 6-month-old PS1/APP and WT mice; PS1/APP mice had significantly higher AT8 levels than to age-matched WT mice $(n=6$, $p<0.05$ ). b The presence of AT8-positive structures around plaques (asterisk) was observed from 4 months of age. c1-c4 and d1-d4. Double-label immunofluorescence confocal laser microscopy of APP (green) and AT8 (red) in 6-month-old transgenic mice showing the presence of phospho-tau in some APP-positive dystrophic neurites. e Immunohistochemistry for cofilin revealed the presence of actin-

cofilin rods around plaques (asterisk) from 4 months of age. A higher magnification detail of a rod is shown in the inset. f1-f3 Confocal laser microscopy with double APP/cofilin labeling revealed the presence of cofilin within some APP-positive neurites. g Quantitative Western blotting for heavy chain kinesin-1 and dynein in 6-month-old PS1/APP and WT mice hippocampus. PS1/APP mice were found to have significantly lower kinesin-1 and dynein levels than agematched WT mice $(n=8, p<0.05)$. Scale bars $\mathbf{b} 100 \mu \mathrm{m}, \mathbf{c 1}-\mathbf{c} 3$ $5 \mu \mathrm{m}, \mathbf{c 4} 2.5 \mu \mathrm{m}, \mathbf{d 1}-\mathbf{d} 37.5 \mu \mathrm{m}, \mathbf{d 4} 2.5 \mu \mathrm{m}$, e $50 \mu \mathrm{m}$ (inset $7 \mu \mathrm{m}$ ), f1-f3 $7.5 \mu \mathrm{m}$ 

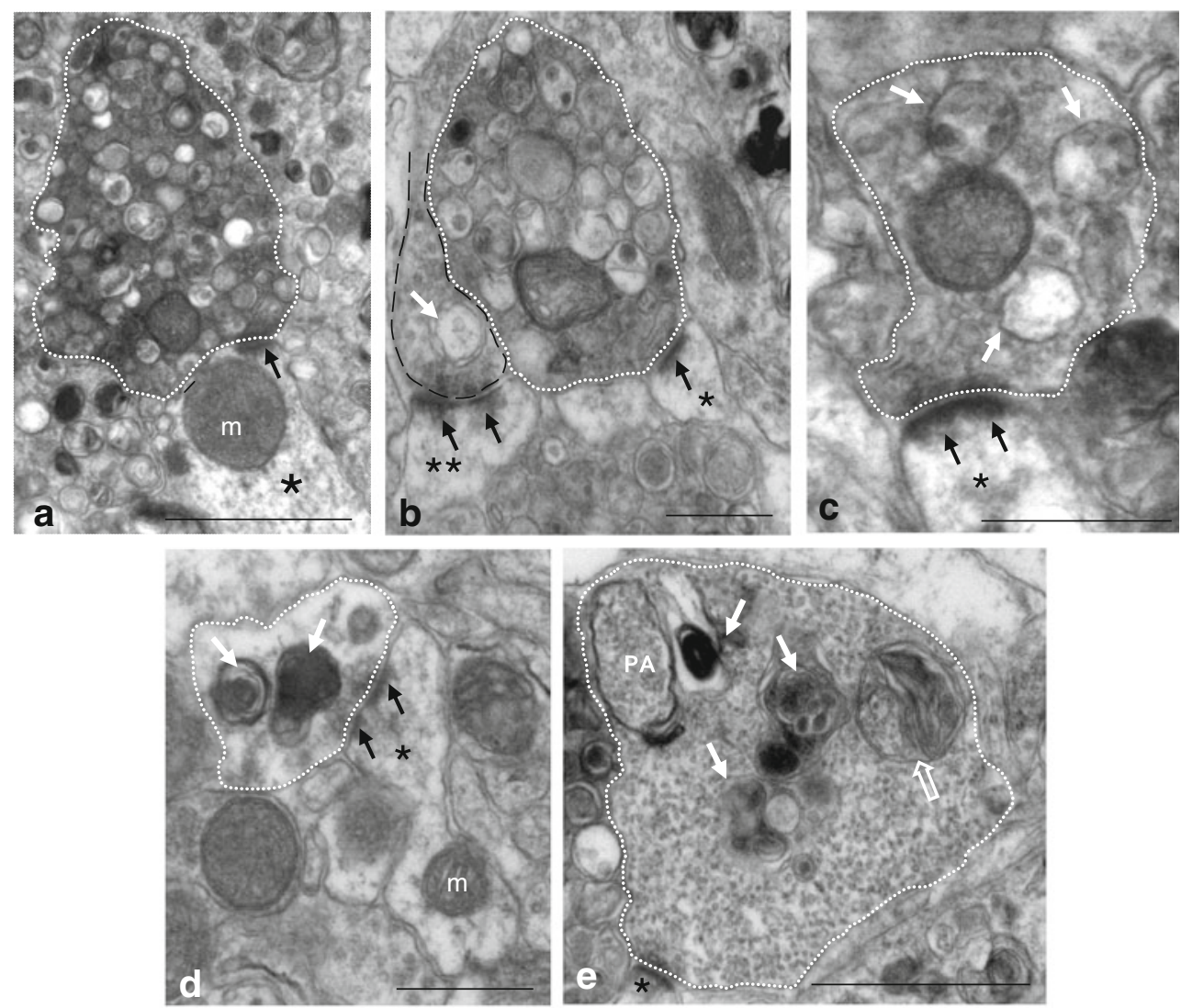

f
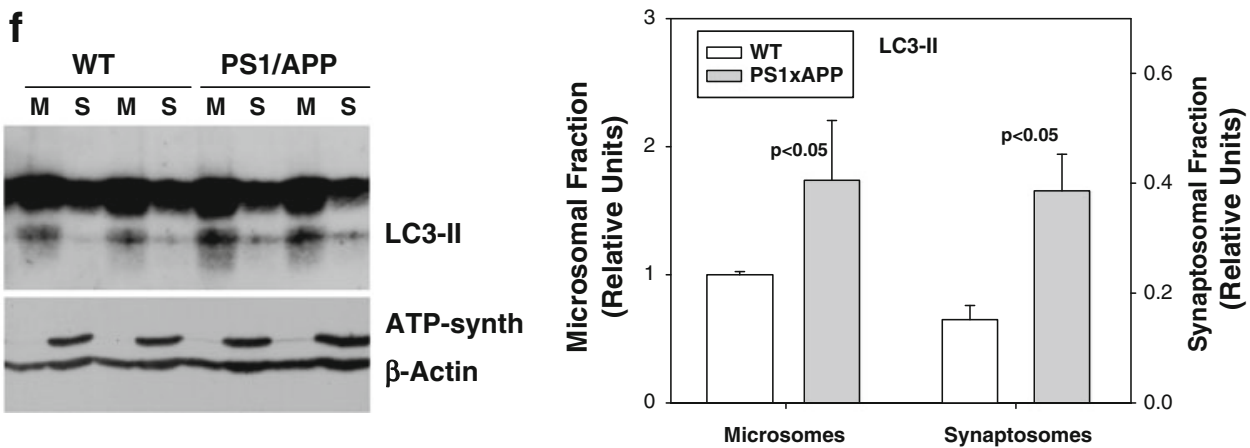

Fig. 6 Autophagy pathology correlates with aberrant presynaptic terminals. a-e Transmission electron microscopy images of aberrant axon terminals (outlined with white lines) containing autophagic vesicles and making asymmetric contact with normal postsynaptic elements (asterisks the postsynaptic densities are indicated with black arrows). a and b Severely disrupted presynaptic terminals (white lines) containing numerous autophagic vesicles and apparently no synaptic vesicles. A presynaptic element (outlined with black lines) that contains one autophagy vesicle (indicated with white arrow), near to the active zone is shown in b. c and $\mathbf{d}$ Presynaptic terminals in a probable initial stage of the dystrophy development, were characterized by the accumulation of relatively few autophagic vesicles (white arrows) and many synaptic vesicles. e Presynaptic terminal showing a putative phagophore $(P A)$ engulfing synaptic-like vesicles, as well as an autophagosome containing mitochondria (open arrow) and few

autophagic vesicles containing degraded material (white arrows). f Quantitative immunoblot analyses of the LC3-II form in microsomes and synaptosomes from 6-month-old PS1/APP and WT mice. LC3-II accumulated in both microsomal and synaptosomal fractions of WT and PS1/APP hippocampus, though a predominant localization was found in the microsomal fraction. The levels of LC3-II in WT synaptosomes were extremely low, as compared with microsomes (please note the different scale bars). Although in PS1/APP most LC3-II was also present in microsomes, the relative accumulation was more patent in synaptosomes (as compared with WT). The relative abundance of LC3-II in each fraction was normalized by the values for WT microsomal fractions. ATPsynthase and $\beta$-actin were used as markers of purity in the isolation procedure and protein loading, respectively. $m$ mitochondria. Scale bars a $1 \mu \mathrm{m}, \mathbf{b}-\mathbf{e} 500 \mathrm{~nm}$

(4-6 months) by increased protein levels of the marker of autophagosome formation LC3-II, by specific immunolocalization of LC3 in the axonal dystrophies,

surrounding plaques, and by electron microscopy morphological identification of the AVs that were completely filling the axon and causing the local axonal swelling. 
Fig. 7 Monomeric A $\beta$ accumulates in isolated synaptosomes whereas $\mathrm{A} \beta$ oligomers are present in the plaque periphery and microsomes. a The $\mathrm{A} \beta$ content were analyzed by $6 \mathrm{E} 10$ immunoprecipitation and Western blots (16\%-TrisTricine-SDS-PAGE) in synaptosomal and microsomal fractions from 6-month-old WT and PS1/APP mice (a1) or by 82E1 Western blots after ureaSDS-PAGE (16\%) in PS1/APP synaptosomal fractions (a2). As shown, the monomeric $A \beta$ was predominantly enriched in the synaptosomal fractions (a1). From the different $A \beta$ peptides, the $\mathrm{A} \beta 42$ was the predominant form found in synaptosomes (a2). b Oligomeric $\mathrm{A} \beta$ peptides were localized at the periphery of the plaques by immunohistochemistry with the A11 antibody. Inset shows a higher magnification of an immunostained amyloid plaque. c The presence of oligomeric $A \beta$ in extracellular soluble fractions (S1), synaptosomes and microsomes was assessed by immunoprecipitation experiments, using the A11 antibody, and Western blots (6E10) using 12\%-Tris-GlycineSDS-PAGE. In agreement with a putative plaque origin, $A \beta$ oligomers (57, 90 and $96 \mathrm{kDa})$ were identified in S1 fractions. d Representative Western blot of WT and PS1/APP microsomal fractions using the mAb 82E1. As shown, $A \beta$ oligomers (ranging $\mathrm{Mr}$ 30-60 kDa) were clearly identified using this antibody. CA1-CA3 subfields of the hippocampus proper, $D G$ dentate gyrus. Scale bars b $250 \mu \mathrm{m}$ (inset $25 \mu \mathrm{m}$ ) a
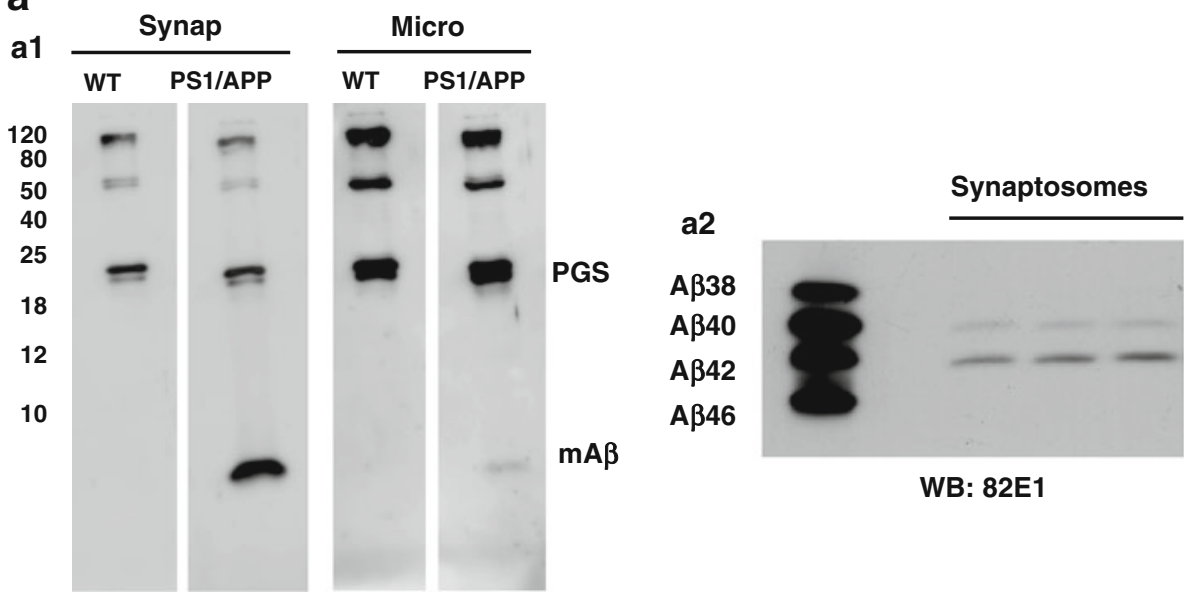

WB: 82E1

WB: 6E10

b

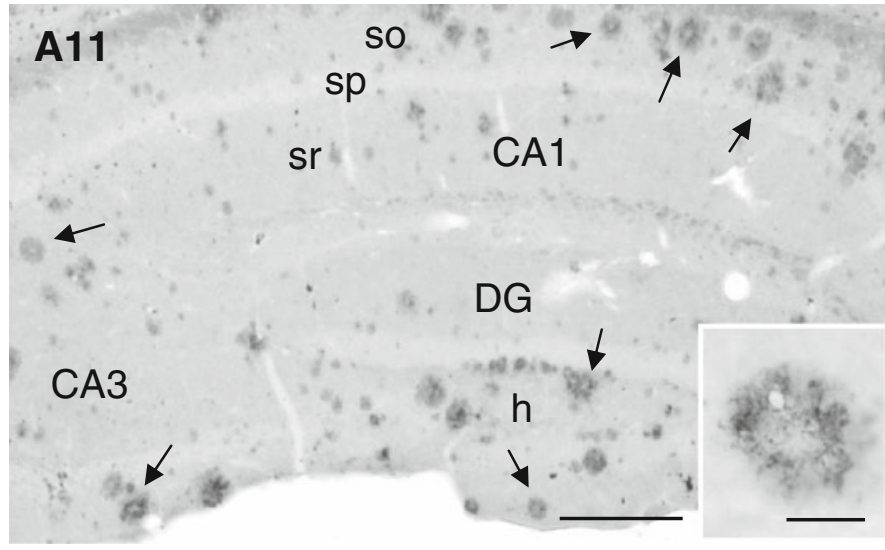

C

IP: A11

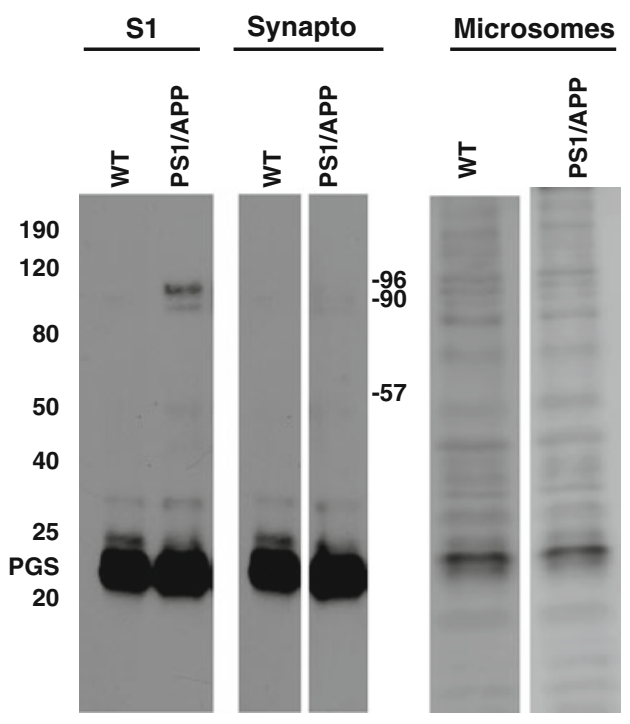

WB: $6 \mathrm{E} 10$ d

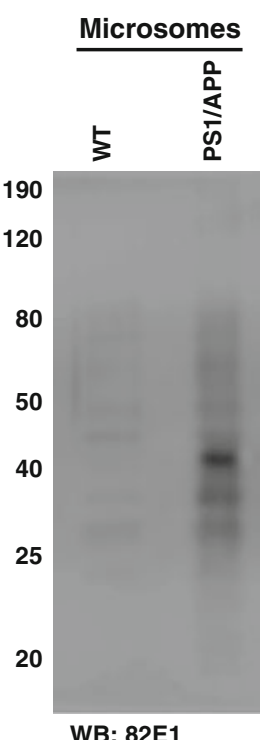

This agreement between different mice models and $\mathrm{AD}$ cases strongly support the involvement of autophagy in the development of AD.
Intact microtubules are needed for $\mathrm{AVs}$ transport and their clearance, facilitating fusion between autophagosomes and late endosomal and lysosomal compartments 
thus preventing the accumulation of immature $A V s$ in neurites $[11,33,36]$. Supporting the possible involvement of microtubule destabilization in $\mathrm{AD}$ pathogenesis, it has been recently reported [31] that natural oligomeric $A \beta$, isolated from $\mathrm{AD}$ patients, disrupted the microtubule cytoskeleton and caused neuritic dystrophy (see also [67]). This effect seemed to be mediated by tau hyperphosphorylation at Ser202/Ser205 (AT8 epitope). In fact, tau reduction prevents $\mathrm{A} \beta$-induced impairment of axonal transport [63]. Interestingly, it has been just reported that disease-associated changes in tau conformation inhibit kinesin-dependent axonal transport by modulating axonal phosphotransferases [32]. In line with this our data indicated the existence of early defective axonal transport within dystrophic axons in our transgenic model. These dystrophic neurites displayed tau phosphorylation (AT8positive) and actin-cofilin rods, and both alterations are known to alter cytoskeletal dynamics in neurons (revised in [6]). Moreover, both the kinesin-1 and dynein levels were significantly reduced and, although non-significantly at 6 months of age, neurofilaments ( $\mathrm{H}$ and $\mathrm{M}$ chains) were phosphorylated (not shown). Taken together, our current results suggest that early tau phosphorylation produced axonal transport defects leading to AV accumulation and neurite pathology.

A relevant issue is the pathogenic signal behind this tau and transport dysregulation. The density and size of the neuritic plaques in our transgenic model increased in an agedependent manner while the number of the associated dystrophic neurites was strongly related to the plaque size. The relationship between formation of extracellular amyloid deposits and their associated dystrophies remains elusive. Whereas some authors have proposed that the appearance of dystrophic neurites precedes plaque formation [2, 23, 27, 56], a recent study using in vivo multiphoton imaging showed that dystrophies develop following amyloid deposition [42]. Supporting the later notion, in our AD model the onset of dystrophic neurites occurred in parallel to the formation of amyloid plaques, and most (if not all) dystrophic neurites were associated with $\mathrm{A} \beta$ plaques. Furthermore, we have also observed plaque-associated axonal dystrophies in GABAergic and cholinergic neurons ([5, 44, 51]; this work, see Fig. $4 \mathrm{~g}$, and unpublished results). These neurons did not express the transgenic APP and, consequently, did not accumulate intracellular $\mathrm{A} \beta[5,44,51]$. Thus, the formation of the dystrophic neurites in these neurons might be induced by extracellular $A \beta$, in close proximity to plaques. In contrast, the high content of APP within the dystrophic neurites suggests a possible direct role of such malformations in plaque formation. Increasing evidence implicates axons as an important source of extracellular amyloid deposits [68] and synaptic activation promotes amyloid secretion, whereas chronic reduction of synaptic activity was found to reduce plaque loading in an AD transgenic mouse model [15, 16, 60]. Interestingly, in our transgenic model, hippocampal plaque deposition is tightly linked to axonal pathways, as seen by simultaneous Congo red staining and neurofilament immunostaining. This agrees with the idea of $A \beta$ released by axonal terminals and the consequent formation of extracellular deposits. In any case, the two processes, plaque induction of neuritic changes and a contribution of dystrophic neurites to $A \beta$ deposition are not mutually exclusive and could occur concomitantly.

The soluble forms of oligomeric amyloid peptides are of special interest to play a significant role in AD pathology. Soluble A $\beta$ oligomers have been shown to induce an increase in tau hyperphosphorylation $[17,31]$ as well as severe axonal transport failure and disruption of organelle trafficking through GSK3-beta signalling (manuscript in preparation;[18, 31]). Furthermore, we have recently reported [30] the involvement of natural and synthetic A $\beta$ oligomers in the activation of GSK3-beta and tau phosphorylation. Thus, it is tempting to speculate that $A \beta$ oligomers, acting through a yet unidentified mediator, caused the interruption of axonal transport, accumulation of vesicles and axonal dystrophy.

The extracellular or intracellular origin of these soluble amyloid oligomers has not yet been well defined. In relation to this, as recently reported in AD cases [55], the results we report here demonstrate that PS1/APP synaptosomes accumulate $A \beta$ peptides $\left(A \beta_{42}\right.$ the most abundant). However, these $\mathrm{A} \beta$ peptides were in monomeric form and no oligomers were detected at this age. On the other hand, we also identified, by immunogold labeling, the presence of APP and $A \beta_{42}$ in AVs which agrees with the participation of the autophagic compartments in APP processing and A $\beta$ production, as described by others $[65,66]$. We also found that AVs were mainly accumulated in the microsomal fractions and, although the $A \beta$ seemed to be produced principally in the synaptosomes, $A \beta$ oligomers were identified in these microsomal fractions. These results provide evidence for the idea that the AVs, within the axonal dystrophies, are a possible compartment for $A \beta$ oligomerization. On the other hand, taken together, the close temporal and spatial association between amyloid plaques and dystrophic neurites, the presence of A11-positive $A \beta$ oligomers in the plaque periphery and the $A \beta$ oligomers in the soluble fractions suggested that plaques also might be a source of the $A \beta$ oligomers that could induce neuritic damage. In support of this idea, amyloid fibrils, major component of amyloid plaques, can be destabilized and easily reverted to soluble and highly toxic $\mathrm{A} \beta$ aggregates by biological lipids that are present in the brain [38] and, as mentioned above, GABAergic cells (that did not express hAPP and did not accumulated $A \beta$ peptides) also displayed axonal 
dystrophies. In any case, further experiments are needed to clarify this point.

A further significant result was the identification by electron microscopy of dystrophic axon terminals that were making contact with morphologically normal postsynaptic elements. These abnormal presynaptic boutons contained numerous $\mathrm{AVs}$ and were observed to have a low content in synaptic vesicles. These results were confirmed by LC3-II Western blots using synaptosomes isolated from the hippocampus of 6-month-old PS1/APP mice. Although we cannot rule out a direct effect of soluble $A \beta$ on these presynaptic terminals, their relative distance from $A \beta$ plaques (between 10 and $30 \mu \mathrm{m}$ ) together with the low soluble $A \beta$ content at this age, suggested that $\mathrm{AV}$ accumulation might reflect the axonal transport defects at dystrophies. These altered synaptic terminals may represent one of the initial pathogenic steps of synaptic loss leading to early deficits in synaptic transmission and plasticity.

As early memory loss in $\mathrm{AD}$ is increasingly attributed to synaptic failure, we can conclude that this PS1/APP transgenic model shows, at young ages and in absence of pyramidal degeneration, a presynaptic pathology progression that may closely resemble the pre-clinical or early stages of human $\mathrm{AD}$.

Acknowledgments This work was supported by grants PS09/00099 (to A.G.), PS09/00151 (to J.V.), PS09/00848 (to D.R.) and PS09/ 00376 (to A.J.J.) from Fondo de Investigación Sanitaria (FIS)-Instituto de Salud Carlos III, Spain. Financial support was also received from Junta de Andalucía grants SAS P09/496 (to A.G.) and CTS-4795 (to J.V.). R.S.V., V.D.C. and S.J. were the recipients of a contract from CIBERNED. E.S.M. and L.T.E. held a PhD fellowship from Spain FPU program. We thank Mercedes Aneiros for her excellent technical assistance and Mario Soriano for his help with the transmission electron microscope.

Open Access This article is distributed under the terms of the Creative Commons Attribution Noncommercial License which permits any noncommercial use, distribution, and reproduction in any medium, provided the original author(s) and source are credited.

\section{References}

1. Adalbert R, Nogradi A, Babetto E et al (2009) Severely dystrophic axons at amyloid plaques remain continuous and connected to viable cell bodies. Brain 132:402-416

2. Arendt T (2005) Alzheimer's disease as a disorder of dynamic brain self-organization. Prog Brain Res 147:355-378

3. Arendt T (2009) Synaptic degeneration in Alzheimer's disease. Acta Neuropathol 118:167-179

4. Ashe KH, Zahs KR (2010) Probing the biology of Alzheimer's disease in mice. Neuron 66:631-645

5. Baglietto-Vargas D, Moreno-Gonzalez I, Sanchez-Varo R et al (2010) Calretinin interneurons are early targets of extracellular amyloid-beta pathology in PS1/AbetaPP Alzheimer mice hippocampus. J Alzheimers Dis 21:119-132
6. Bamburg JR, Bloom GS (2009) Cytoskeletal pathologies of Alzheimer disease. Cell Motil Cytoskelet 66:635-649

7. Bell KF, Claudio CA (2006) Altered synaptic function in Alzheimer's disease. Eur J Pharmacol 545:11-21

8. Bell KF, de Kort GJ, Steggerda S et al (2003) Structural involvement of the glutamatergic presynaptic boutons in a transgenic mouse model expressing early onset amyloid pathology. Neurosci Lett 353:143-147

9. Bertoni-Freddari C, Fattoretti P, Solazzi M et al (2003) Neuronal death versus synaptic pathology in Alzheimer's disease. Ann NY Acad Sci 1010:635-638

10. Blanchard V, Moussaoui S, Czech C et al (2003) Time sequence of maturation of dystrophic neurites associated with Abeta deposits in APP/PS1 transgenic mice. Exp Neurol 184:247263

11. Boland B, Kumar A, Lee S et al (2008) Autophagy induction and autophagosome clearance in neurons: relationship to autophagic pathology in Alzheimer's disease. J Neurosci 28:6926-6937

12. Caballero C, Jimenez S, Moreno-Gonzalez I et al (2007) Interindividual variability in the expression of the mutated form of hPS1M146L determined the production of Abeta peptides in the PS1xAPP transgenic mice. J Neurosci Res 85:787-797

13. Castellani RJ, Rolston RK, Smith MA (2010) Alzheimer disease. Dis Mon 56:484-546

14. Chishti MA, Yang DS, Janus C et al (2001) Early-onset amyloid deposition and cognitive deficits in transgenic mice expressing a double mutant form of amyloid precursor protein 695. J Biol Chem 276:21562-21570

15. Cirrito JR, Kang JE, Lee J et al (2008) Endocytosis is required for synaptic activity-dependent release of amyloid-beta in vivo. Neuron 58:42-51

16. Cirrito JR, Yamada KA, Finn MB et al (2005) Synaptic activity regulates interstitial fluid amyloid-beta levels in vivo. Neuron 48:913-922

17. De Felice FG, Wu D, Lambert MP et al (2008) Alzheimer's disease-type neuronal tau hyperphosphorylation induced by Abeta oligomers. Neurobiol Aging 29:1334-1347

18. Decker H, Lo KY, Unger SM, Ferreira ST, Silverman MA (2010) Amyloid-beta peptide oligomers disrupt axonal transport through an NMDA receptor-dependent mechanism that is mediated by glycogen synthase kinase 3beta in primary cultured hippocampal neurons. J Neurosci 30:9166-9171

19. DeKosky ST, Scheff SW (1990) Synapse loss in frontal cortex biopsies in Alzheimer's disease: correlation with cognitive severity. Ann Neurol 27:457-464

20. Delatour B, Blanchard V, Pradier L, Duyckaerts C (2004) Alzheimer pathology disorganizes cortico-cortical circuitry: direct evidence from a transgenic animal model. Neurobiol Dis $16: 41-47$

21. Dickson TC, King CE, McCormack GH, Vickers JC (1999) Neurochemical diversity of dystrophic neurites in the early and late stages of Alzheimer's disease. Exp Neurol 156:100-110

22. Dickson TC, Vickers JC (2001) The morphological phenotype of beta-amyloid plaques and associated neuritic changes in Alzheimer's disease. Neuroscience 105:99-107

23. Fiala JC (2007) Mechanisms of amyloid plaque pathogenesis. Acta Neuropathol 114:551-571

24. Geddes JW, Anderson KJ, Cotman CW (1986) Senile plaques as aberrant sprout-stimulating structures. Exp Neurol 94:767-776

25. Geddes JW, Monaghan DT, Cotman CW et al (1985) Plasticity of hippocampal circuitry in Alzheimer's disease. Science 230:1179-1181

26. Gomez-Isla T, Spires T, de Calignon A, Hyman BT (2008) Neuropathology of Alzheimer's disease. Handb Clin Neurol 89:233-243 
27. Gouras GK, Almeida CG, Takahashi RH (2005) Intraneuronal Abeta accumulation and origin of plaques in Alzheimer's disease. Neurobiol Aging 26:1235-1244

28. Hussain I (2010) APP transgenic mouse models and their use in drug discovery to evaluate amyloid-lowering therapeutics. CNS Neurol Disord Drug Targets 9:395-402

29. Jimenez S, Baglietto-Vargas D, Caballero C et al (2008) Inflammatory response in the hippocampus of PS1M146L/ APP751SL mouse model of Alzheimer's disease: age-dependent switch in the microglial phenotype from alternative to classic. J Neurosci 28:11650-11661

30. Jimenez S, Torres M, Vizuete M et al (2011) Age-dependent accumulation of soluble Abeta oligomers reverses the neuroprotective effect of sAPPalpha by modulating PI3K/Akt-GSK-3beta pathway in Alzheimer mice model. J Biol Chem 286:18414-18425

31. Jin M, Shepardson N, Yang T et al (2011) Soluble amyloid \{beta\}-protein dimers isolated from Alzheimer cortex directly induce Tau hyperphosphorylation and neuritic degeneration. Proc Natl Acad Sci USA 108:5819-5824

32. Kanaan NM, Morfini GA, Lapointe NE et al (2011) Pathogenic forms of tau inhibit kinesin-dependent axonal transport through a mechanism involving activation of axonal phosphotransferases. J Neurosci 31:9858-9868

33. Kochl R, Hu XW, Chan EY, Tooze SA (2006) Microtubules facilitate autophagosome formation and fusion of autophagosomes with endosomes. Traffic 7:129-145

34. Koenigsknecht-Talboo J, Meyer-Luehmann M, Parsadanian M et al (2008) Rapid microglial response around amyloid pathology after systemic anti-Abeta antibody administration in PDAPP mice. J Neurosci 28:14156-14164

35. Koffie RM, Meyer-Luehmann M, Hashimoto T et al (2009) Oligomeric amyloid beta associates with postsynaptic densities and correlates with excitatory synapse loss near senile plaques. Proc Natl Acad Sci USA 106:4012-4017

36. Kovacs AL, Reith A, Seglen PO (1982) Accumulation of autophagosomes after inhibition of hepatocytic protein degradation by vinblastine, leupeptin or a lysosomotropic amine. Exp Cell Res 137:191-201

37. Lee JH, Yu WH, Kumar A et al (2010) Lysosomal proteolysis and autophagy require presenilin 1 and are disrupted by Alzheimer-related PS1 mutations. Cell 141:1146-1158

38. Martins IC, Kuperstein I, Wilkinson H et al (2008) Lipids revert inert Abeta amyloid fibrils to neurotoxic protofibrils that affect learning in mice. EMBO J 27:224-233

39. Masliah E, Alford M, Adame A et al (2003) Abeta1-42 promotes cholinergic sprouting in patients with $\mathrm{AD}$ and Lewy body variant of AD. Neurology 61:206-211

40. Masliah E, Hansen L, Albright T, Mallory M, Terry RD (1991) Immunoelectron microscopic study of synaptic pathology in Alzheimer's disease. Acta Neuropathol 81:428-433

41. Masliah E, Mallory M, Alford M et al (2001) Altered expression of synaptic proteins occurs early during progression of Alzheimer's disease. Neurology 56:127-129

42. Meyer-Luehmann M, Spires-Jones TL, Prada C et al (2008) Rapid appearance and local toxicity of amyloid-beta plaques in a mouse model of Alzheimer's disease. Nature 451:720724

43. Mirra SS, Heyman A, McKeel D et al (1991) The Consortium to Establish a Registry for Alzheimer's Disease (CERAD). Part II. Standardization of the neuropathologic assessment of Alzheimer's disease. Neurology 41:479-486

44. Moreno-Gonzalez I, Baglietto-Vargas D, Sanchez-Varo R et al (2009) Extracellular amyloid-beta and cytotoxic glial activation induce significant entorhinal neuron loss in young PS1(M146L)/ APP(751SL) mice. J Alzheimers Dis 18:755-776
45. Nimmrich V, Ebert U (2009) Is Alzheimer's disease a result of presynaptic failure? Synaptic dysfunctions induced by oligomeric beta-amyloid. Rev Neurosci 20:1-12

46. Nixon RA (2007) Autophagy, amyloidogenesis and Alzheimer disease. J Cell Sci 120:4081-4091

47. Nixon RA, Wegiel J, Kumar A et al (2005) Extensive involvement of autophagy in Alzheimer disease: an immuno-electron microscopy study. J Neuropathol Exp Neurol 64:113-122

48. Nixon RA, Yang DS (2011) Autophagy failure in Alzheimer's disease-locating the primary defect. Neurobiol Dis 43:38-45

49. Noda-Saita K, Terai K, Iwai A et al (2004) Exclusive association and simultaneous appearance of congophilic plaques and AT8positive dystrophic neurites in Tg2576 mice suggest a mechanism of senile plaque formation and progression of neuritic dystrophy in Alzheimer's disease. Acta Neuropathol 108:435-442

50. Perl DP (2010) Neuropathology of Alzheimer's disease. Mt Sinai J Med 77:32-42

51. Ramos B, Baglietto-Vargas D, Del Rio JC et al (2006) Early neuropathology of somatostatin/NPY GABAergic cells in the hippocampus of a PS1xAPP transgenic model of Alzheimer's disease. Neurobiol Aging 27:1658-1672

52. Scheff SW, Price DA, Schmitt FA, DeKosky ST, Mufson EJ (2007) Synaptic alterations in CA1 in mild Alzheimer disease and mild cognitive impairment. Neurology 68:1501-1508

53. Scheff SW, Price DA, Schmitt FA, Mufson EJ (2006) Hippocampal synaptic loss in early Alzheimer's disease and mild cognitive impairment. Neurobiol Aging 27:1372-1384

54. Selkoe DJ (2002) Alzheimer's disease is a synaptic failure. Science 298:789-791

55. Sokolow S, Henkins KM, Bilousova T et al (2011) AD synapses contain abundant Abeta monomer and multiple soluble oligomers, including a 56-kDa assembly. Neurobiol Aging. doi: 10.1016/j.neurobiolaging.2011.05.011

56. Stokin GB, Lillo C, Falzone TL et al (2005) Axonopathy and transport deficits early in the pathogenesis of Alzheimer's disease. Science 307:1282-1288

57. Su JH, Cummings BJ, Cotman CW (1993) Identification and distribution of axonal dystrophic neurites in Alzheimer's disease. Brain Res 625:228-237

58. Su JH, Cummings BJ, Cotman CW (1998) Plaque biogenesis in brain aging and Alzheimer's disease. II. Progressive transformation and developmental sequence of dystrophic neurites. Acta Neuropathol 96:463-471

59. Sze CI, Troncoso JC, Kawas C et al (1997) Loss of the presynaptic vesicle protein synaptophysin in hippocampus correlates with cognitive decline in Alzheimer disease. J Neuropathol Exp Neurol 56:933-944

60. Tampellini D, Gouras GK (2010) Synapses, synaptic activity and intraneuronal abeta in Alzheimer's disease. Front Aging Neurosci 2:13

61. Terry RD, Masliah E, Salmon DP et al (1991) Physical basis of cognitive alterations in Alzheimer's disease: synapse loss is the major correlate of cognitive impairment. Ann Neurol 30:572-580

62. Vitorica J, Satrustegui J (1986) Involvement of mitochondria in the age-dependent decrease in calcium uptake of rat brain synaptosomes. Brain Res 378:36-48

63. Vossel KA, Zhang K, Brodbeck J et al (2010) Tau reduction prevents Abeta-induced defects in axonal transport. Science 330:198

64. Woodhouse A, Vickers JC, Adlard PA, Dickson TC (2009) Dystrophic neurites in TgCRND8 and Tg2576 mice mimic human pathological brain aging. Neurobiol Aging 30:864-874

65. Yang DS, Stavrides P, Mohan PS et al (2011) Reversal of autophagy dysfunction in the TgCRND8 mouse model of Alzheimer's disease ameliorates amyloid pathologies and memory deficits. Brain 134:258-277 
66. Yu WH, Kumar A, Peterhoff C et al (2004) Autophagic vacuoles are enriched in amyloid precursor protein-secretase activities: implications for beta-amyloid peptide over-production and localization in Alzheimer's disease. Int J Biochem Cell Biol 36:2531-2540

67. Zempel H, Thies E, Mandelkow E, Mandelkow EM (2010) Abeta oligomers cause localized $\mathrm{Ca}(2+)$ elevation, missorting of endogenous Tau into dendrites, Tau phosphorylation, and destruction of microtubules and spines. J Neurosci 30:11938-11950

68. Zhang XM, Cai Y, Xiong K et al (2009) Beta-secretase-1 elevation in transgenic mouse models of Alzheimer's disease is associated with synaptic/axonal pathology and amyloidogenesis: implications for neuritic plaque development. Eur J Neurosci 30:2271-2283 